\title{
Optical monitoring of the gravitationally lensed quasar Q2237+0305 from APO between June 1995 and January 1998 ${ }^{\star}$
}

\author{
R. W. Schmidt ${ }^{1,2}$, T. Kundić ${ }^{3}$, U.-L. Pen ${ }^{4}$, E. L. Turner ${ }^{5}$, J. Wambsganss ${ }^{2}$, L. E. Bergeron ${ }^{6}$, W. N. Colley ${ }^{7}$, \\ C. Corson ${ }^{8}$, N. C. Hastings ${ }^{9}$, T. Hoyes ${ }^{10}$, D. C. Long ${ }^{9}$, K. A. Loomis ${ }^{9}$, S. Malhotra ${ }^{11, \star \star}$, \\ J. E. Rhoads ${ }^{6}$, and K. Z. Stanek ${ }^{12}$ \\ 1 Institute of Astronomy, Madingley Road, Cambridge CB3 0HA, UK \\ 2 Universität Potsdam, Institut für Physik, Am Neuen Palais 10, 14469 Potsdam, Germany \\ ${ }^{3}$ Renaissance Technologies, 600 Route 25A, East Setauket, NY 11733, USA \\ ${ }^{4}$ Canadian Institute of Theoretical Astrophysics, 60 St. George St., Toronto, M5S 3H8, Canada \\ ${ }^{5}$ Princeton University Observatory, Peyton Hall, Princeton, NJ 08544, USA \\ 6 Space Telescope Science Institute, 3700 San Martin Drive, Baltimore, MD 21218, USA \\ 7 Lincoln Laboratory, Massachusetts Institute of Technology, 244 Wood Street, Lexington, MA 02420, USA \\ 8 National Optical Astronomy Observatory, PO Box 26732, Tucson, AZ 85726, USA \\ 9 Apache Point Observatory, 2001 Apache Point Rd, Sunspot NM 88349, USA \\ 10 Mesa Community College, 1833 West Southern Ave., Mesa, Arizona 85202-4866, USA \\ 11 Johns Hopkins University, Charles and 34th Street, Bloomberg Center, Baltimore, MD 21210, USA \\ 12 Harvard-Smithsonian Center for Astrophysics, 60 Garden Street, Cambridge, MA 02138, USA
}

Received 31 January 2002 / Accepted 20 June 2002

\begin{abstract}
We present a data set of images of the gravitationally lensed quasar Q2237+0305, that was obtained at the Apache Point Observatory (APO) between June 1995 and January 1998. Although the images were taken under variable, often poor seeing conditions and with coarse pixel sampling, photometry is possible for the two brighter quasar images A and B with the help of exact quasar image positions from HST observations. We obtain a light curve with 73 data points for each of the images A and B. There is evidence for a long ( $\gtrsim 100$ day) brightness peak in image A in 1996 with an amplitude of about 0.4 to $0.5 \mathrm{mag}$ (relative to 1995), which indicates that microlensing has been taking place in the lensing galaxy. Image B does not vary much over the course of the observation period. The long, smooth variation of the light curve is similar to the results from the OGLE monitoring of the system (Woźniak et al. 2000a).
\end{abstract}

Key words. gravitational lensing - dark matter - quasars: individual: Q2237+0305 - cosmology: observations

\section{Introduction}

The quadruple quasar Q2237+0305 was discovered during the Center for Astrophysics (CfA) redshift survey (Huchra et al. 1985). In high-resolution images of the system, four quasar images at a redshift of $z=1.695$ are seen in a cross-like geometry around the core of a barred spiral galaxy with a redshift of $z=0.0394$. Due to its geometry this system is known as the Einstein cross (Schneider et al. 1988; Yee 1988). The quasar images have a separation of about 0.9 arcsec from the galaxy centre.

Send offprint requests to: $\mathrm{R}$. W. Schmidt,

e-mail: rschmidt@ast.cam.ac.uk

* Based on observations obtained with the Apache Point Observatory 3.5-meter telescope, which is owned and operated by the Astrophysical Research Consortium.

$\star \star$ Hubble Fellow.
Very quickly after its discovery it was realized that this system is an ideal case for microlensing studies. The challenge in this system is to measure the brightness of the four images individually with high accuracy. In observations with seeing of larger than about an arcsecond, it is very difficult to disentangle the four quasar images, the galaxy core, and other features of the galaxy (such as the bar-like structure that is situated across the galaxy centre, see Yee 1988, or Schmidt et al. 1998).

The record of semi-regular observations of this system began with the announcement of the first microlensing event by Irwin et al. (1989). Corrigan et al. (1991) published the "initial light curve", that was later augmented by other individual and systematical observations (Pen et al. 1993; Houde \& Racine 1994). In Wambsganss (1992) it was emphasized that without frequent sampling, it would be difficult to extract useful information from the microlensing observations. The sample of early observations with good seeing is rather heterogeneous 
regarding the filters chosen, so that the interpretation is made difficult since the filter differences have to be calibrated out.

$\emptyset$ stensen et al. (1996) presented five years of observations of Q2237+0305 from the Nordic Optical Telescope (NOT). In all four images, microlensing variations had then been detected. A rather striking drop of about one magnitude in 1992 (with very large error bars) within $\approx 20$ days had been found by Pen et al. (1993) on the basis of observations made at the Apache Point Observatory. In addition to this photometric evidence, Lewis et al. (1998) found spectroscopic signatures for microlensing of the broad line region of this quasar.

Recently, the OGLE team has presented a light curve (Woźniak et al. 2000a,b) covering about 1200 days between 1997 and 2001. Updated versions of this photometric data set can be looked at www.astro.princeton.edu/ ogle/ ogle2/huchra.html. This data set provided a major step forward, and allows qualitatively new approaches in the analysis of the light curves. The OGLE light curves are very densely sampled and show amazing brightness variations in all four quasar images with high amplitudes of more than one magnitude. Especially image $\mathrm{C}$ shows a dramatic brightness peak of about $1.2 \mathrm{mag}$ in 1999 that was resolved by the OGLE data in beautiful detail.

In Sect. 2 our data set is described. In Sect. 3 we explain and describe the details of the data reduction. In Sect. 4 we present our results. We conclude in Sect. 5 with a discussion.

\section{Q2237+0305 at Apache Point Observatory}

We describe here the reduction and analysis of data that were taken with the $3.5 \mathrm{~m}$ telescope at Apache Point Observatory (APO) from June 1995 to January 1998 as part of the PrincetonAPO lens monitoring program. We restrict ourselves to the $r$-band data. The corresponding CCD has a pixel size of $0.61^{\prime \prime}$. In Table 1, the observation $\log$ is given.

The images were taken under variable, often poor seeing conditions between 1.2" and several arcseconds. We have filtered out frames with seeing worse than $2.4^{\prime \prime}$. The distance between two quasar images is only about three pixels. In these frames, the four quasar images, the galaxy core, and the remaining light from the galaxy are contained within only a dozen or so pixels, which is a fairly coarse sampling compared to the number of sources in this area. In Fig. 1, the centre of a frame obtained on 20 November 1996 under 1.2" seeing is shown. Except for the overall central light concentration, the detailed quasar image- and galaxy structure of this system is not visible.

However, we know the exact quasar image positions relative to the galaxy centre from HST observation in the UV (Blanton et al. 1998) with an accuracy of 5 mas. Moreover, a detailed model of the galaxy light distribution is available (Schmidt 1996) that was obtained from the analysis of HST images as well. With the help of these strong constraints, we are able to measure the brightness of the two brighter quasar images $\mathrm{A}$ and $\mathrm{B}$.

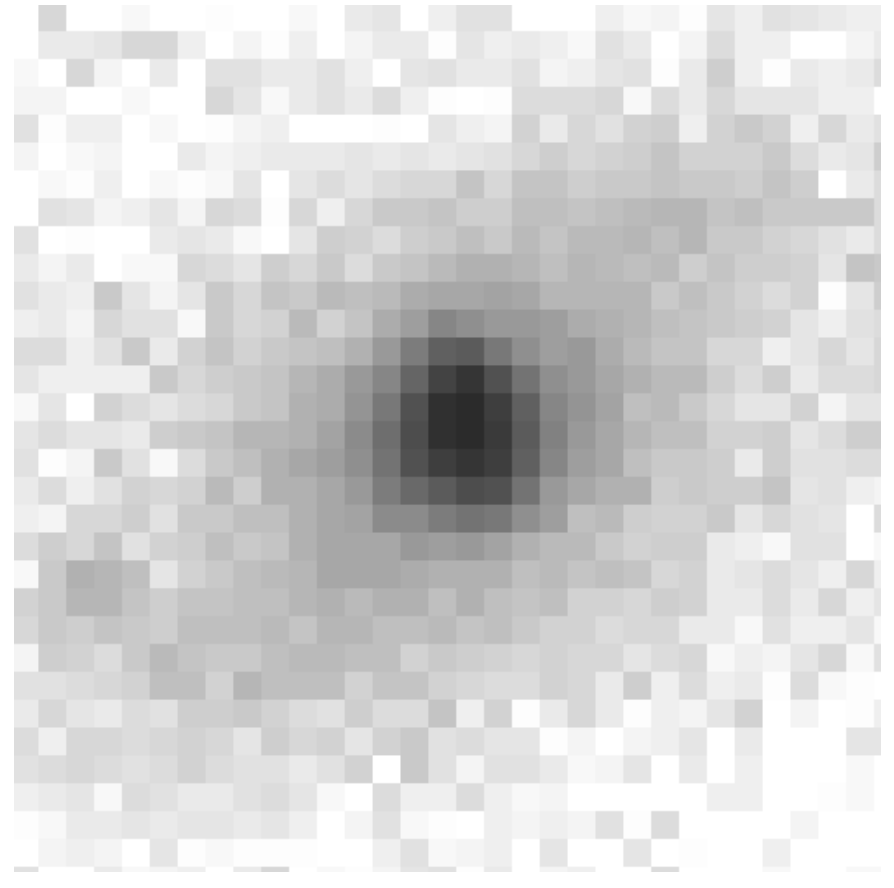

Fig. 1. Image of the central $(18.6 \mathrm{arcsec})^{2}$ of the lensing galaxy $2237+0305$ in the $r$-band. The image was taken at APO on 20 November 1996 under 1.2" seeing. North is down and east is to the left.

\section{Data reduction}

A total of 530 frames of Q2237+0305 were obtained at Apache Point Observatory in the $r$-filter in 73 nights between June 1995 and January 1998 (27 nights in 1995, 33 in 1996, 11 in 1997 and 2 in early 1998). The frames are processed using an automated procedure with the following five steps of the data reduction:

1. Four reference stars in the vicinity of the galaxy were chosen as reference points (see Fig. 2). We picked only the brightest stars in order to be able to determine reliable centroids. These reference stars are registered on each frame using an automated routine;

2. Once the four reference stars are localized, the seeing is determined by fitting a circular Gaussian brightness profile to the reference stars with the IRAF task "imexamine". These four determinations are occasionally different from each other by up to ten percent. We use the seeing of the star closest to the quasars (star \#3) as reference. The distances of stars \#2, \#3 and \#4 from the centre of the galaxy are measured. These distances will be refined in step 5, but we need an approximate input to start the quasar and galaxy fitting procedure in step 4.

The brightest reference star \#2 is used for the absolute flux calibration. In Fig. 3, the difference between the measured magnitudes of stars \#2 and \#3 is depicted as a function of time. The differences plotted in this figure scatter around a median difference of $m_{3}-m_{2}=1.63 \mathrm{mag}$, which is indicated by the dashed line. The error bars are simply determined by Poisson statistics from the flux counts. Except for a few outliers, the data are consistent with our 
Table 1. Observation log of the $r$-band observations of Q2237+0305 at Apache Point Observatory from 1995 to 1998. The table contains the date in yymmdd format, the Julian date $(-2449000)$, the total exposure time of all exposures in a given night, the seeing as determined from reference star 3 in the stacked images, and the magnitudes with $1 \sigma$ errors in parentheses.

\begin{tabular}{|c|c|c|c|c|c|}
\hline date & $\begin{array}{l}\text { Julian } \\
\text { date }\end{array}$ & $\begin{array}{l}\text { total exposure } \\
\text { time per night } \\
\text { (seconds) }\end{array}$ & $\begin{array}{l}\text { seeing } \\
(\operatorname{arcsec})\end{array}$ & $\begin{array}{r}\text { image A } \\
(\mathrm{mag})\end{array}$ & $\begin{array}{r}\text { image B } \\
(\mathrm{mag})\end{array}$ \\
\hline 950602 & 871 & 45 & 2.0 & $17.57(0.15)$ & $17.67(0.17)$ \\
\hline 950606 & 875 & 300 & 2.2 & $17.42(0.20)$ & $17.80(0.13)$ \\
\hline 950616 & 885 & 225 & 1.7 & $17.30(0.10)$ & $17.50(0.10)$ \\
\hline 950620 & 889 & 195 & 2.1 & $17.37(0.12)$ & $17.30(0.11)$ \\
\hline 950624 & 893 & 300 & 1.7 & $17.20(0.14)$ & $17.32(0.13)$ \\
\hline 950625 & 894 & 150 & 1.9 & $17.33(0.16)$ & $17.53(0.14)$ \\
\hline 950628 & 897 & 150 & 1.4 & $17.32(0.13)$ & $17.38(0.10)$ \\
\hline 950702 & 901 & 195 & 2.0 & $17.29(0.15)$ & $17.47(0.14)$ \\
\hline 950704 & 903 & 420 & 1.7 & $17.32(0.10)$ & $17.74(0.12)$ \\
\hline 950710 & 909 & 390 & 1.7 & $17.20(0.13)$ & $17.53(0.14)$ \\
\hline 950712 & 911 & 175 & 1.8 & $16.92(0.12)$ & $17.63(0.16)$ \\
\hline 950722 & 921 & 525 & 1.6 & $17.25(0.12)$ & $17.68(0.13)$ \\
\hline 950805 & 935 & 390 & 2.4 & $17.31(0.21)$ & $17.72(0.17)$ \\
\hline 950819 & 949 & 60 & 2.0 & $17.28(0.17)$ & $17.45(0.14)$ \\
\hline 950821 & 951 & 255 & 2.1 & $17.20(0.17)$ & $17.51(0.16)$ \\
\hline 950823 & 953 & 180 & 2.2 & $17.24(0.15)$ & $17.75(0.12)$ \\
\hline 950825 & 955 & 330 & 2.2 & $17.36(0.16)$ & $17.55(0.15)$ \\
\hline 950827 & 957 & 375 & 2.2 & $17.34(0.19)$ & $17.44(0.16)$ \\
\hline 950911 & 972 & 270 & 1.6 & $17.24(0.12)$ & $17.55(0.13)$ \\
\hline 950923 & 984 & 270 & 1.9 & $17.07(0.12)$ & $17.73(0.16)$ \\
\hline 950925 & 986 & 150 & 1.8 & $17.02(0.10)$ & $17.77(0.16)$ \\
\hline 950929 & 990 & 510 & 1.8 & $17.16(0.12)$ & $17.53(0.14)$ \\
\hline 951001 & 992 & 570 & 1.7 & $17.25(0.13)$ & $17.71(0.14)$ \\
\hline 951003 & 994 & 540 & 1.9 & $17.17(0.12)$ & $17.51(0.13)$ \\
\hline 951015 & 1006 & 300 & 1.7 & $17.08(0.10)$ & $17.68(0.13)$ \\
\hline 951017 & 1008 & 120 & 1.6 & $17.13(0.09)$ & $17.88(0.14)$ \\
\hline 960717 & 1282 & 180 & 1.4 & $17.19(0.10)$ & $17.94(0.12)$ \\
\hline 960719 & 1284 & 210 & 1.4 & $17.20(0.12)$ & $17.66(0.12)$ \\
\hline 960721 & 1286 & 270 & 1.3 & $17.24(0.08)$ & $17.93(0.11)$ \\
\hline 960723 & 1288 & 210 & 1.4 & $17.00(0.10)$ & $17.79(0.12)$ \\
\hline 960728 & 1293 & 150 & 1.3 & $17.08(0.10)$ & $17.76(0.10)$ \\
\hline 960804 & 1300 & 240 & 1.6 & $17.33(0.10)$ & $17.71(0.12)$ \\
\hline 960806 & 1302 & 330 & 1.6 & $16.97(0.09)$ & $17.71(0.13)$ \\
\hline 960810 & 1306 & 270 & 1.4 & $17.19(0.09)$ & $17.80(0.11)$ \\
\hline 960812 & 1308 & 270 & 1.4 & $16.99(0.09)$ & $17.86(0.13)$ \\
\hline 960814 & 1310 & 360 & 1.8 & $16.79(0.13)$ & $17.64(0.16)$ \\
\hline 960818 & 1314 & 270 & 1.4 & $17.00(0.11)$ & $17.72(0.13)$ \\
\hline 960903 & 1330 & 180 & 1.4 & $17.13(0.09)$ & $17.66(0.10)$ \\
\hline 960907 & 1334 & 90 & 1.5 & $16.97(0.09)$ & $17.75(0.13)$ \\
\hline 960909 & 1336 & 90 & 1.8 & $16.55(0.11)$ & $18.04(0.20)$ \\
\hline 960915 & 1342 & 180 & 2.5 & $16.77(0.19)$ & $17.95(0.22)$ \\
\hline 960917 & 1344 & 180 & 1.9 & $16.70(0.14)$ & $17.73(0.18)$ \\
\hline 960921 & 1348 & 90 & 1.4 & $16.86(0.10)$ & $17.63(0.13)$ \\
\hline 960927 & 1354 & 270 & 1.9 & $16.79(0.12)$ & $17.82(0.16)$ \\
\hline 960929 & 1356 & 120 & 2.0 & $16.82(0.15)$ & $17.85(0.20)$ \\
\hline 961003 & 1360 & 90 & 2.8 & $16.80(0.20)$ & $18.12(0.22)$ \\
\hline 961007 & 1364 & 180 & 2.3 & $16.76(0.23)$ & $17.89(0.21)$ \\
\hline 961011 & 1368 & 120 & 1.3 & $16.99(0.09)$ & $17.72(0.12)$ \\
\hline 961013 & 1370 & 285 & 1.3 & $17.14(0.07)$ & $17.84(0.10)$ \\
\hline 961018 & 1375 & 60 & 1.9 & $16.86(0.14)$ & $17.48(0.15)$ \\
\hline
\end{tabular}


Table 1. continued.

\begin{tabular}{lcccrc}
\hline \hline date & $\begin{array}{c}\text { Julian } \\
\text { date }\end{array}$ & $\begin{array}{c}\text { total exposure } \\
\text { time per night } \\
\text { (seconds) }\end{array}$ & $\begin{array}{c}\text { seeing } \\
(\operatorname{arcsec})\end{array}$ & $\begin{array}{r}\text { image A } \\
(\mathrm{mag})\end{array}$ & $\begin{array}{r}\text { image B } \\
(\mathrm{mag})\end{array}$ \\
\hline 961030 & 1387 & 120 & 2.0 & $16.68(0.12)$ & $17.81(0.20)$ \\
961101 & 1389 & 240 & 1.5 & $16.86(0.08)$ & $17.72(0.14)$ \\
961103 & 1391 & 120 & 2.3 & $16.92(0.13)$ & $18.04(0.21)$ \\
961107 & 1395 & 150 & 1.3 & $16.98(0.07)$ & $17.88(0.11)$ \\
961109 & 1397 & 90 & 1.3 & $17.14(0.08)$ & $17.97(0.13)$ \\
961111 & 1399 & 90 & 1.3 & $16.94(0.07)$ & $18.02(0.14)$ \\
961120 & 1408 & 510 & 1.4 & $17.00(0.07)$ & $18.03(0.13)$ \\
961124 & 1412 & 210 & 1.7 & $17.17(0.10)$ & $17.69(0.10)$ \\
961126 & 1414 & 630 & 1.7 & $16.94(0.10)$ & $17.93(0.15)$ \\
& & & & & \\
970715 & 1645 & 140 & 1.4 & $17.05(0.12)$ & $17.81(0.15)$ \\
971018 & 1740 & 180 & 1.8 & $17.21(0.14)$ & $17.86(0.16)$ \\
971026 & 1748 & 390 & 1.3 & $17.28(0.06)$ & $18.23(0.10)$ \\
971030 & 1752 & 240 & 1.4 & $17.34(0.13)$ & $17.74(0.13)$ \\
971101 & 1754 & 210 & 1.6 & $17.45(0.14)$ & $17.55(0.12)$ \\
971103 & 1756 & 270 & 1.7 & $17.25(0.15)$ & $17.85(0.16)$ \\
971105 & 1758 & 630 & 2.2 & $17.52(0.16)$ & $17.89(0.14)$ \\
971121 & 1774 & 220 & 1.7 & $17.16(0.11)$ & $17.97(0.16)$ \\
971125 & 1778 & 630 & 1.9 & $17.27(0.20)$ & $17.74(0.18)$ \\
971129 & 1782 & 450 & 1.3 & $17.34(0.09)$ & $17.88(0.12)$ \\
980101 & 1815 & 30 & 1.7 & $17.21(0.10)$ & $17.85(0.14)$ \\
980111 & 1825 & 60 & 2.0 & $17.04(0.15)$ & $17.64(0.14)$ \\
\hline
\end{tabular}

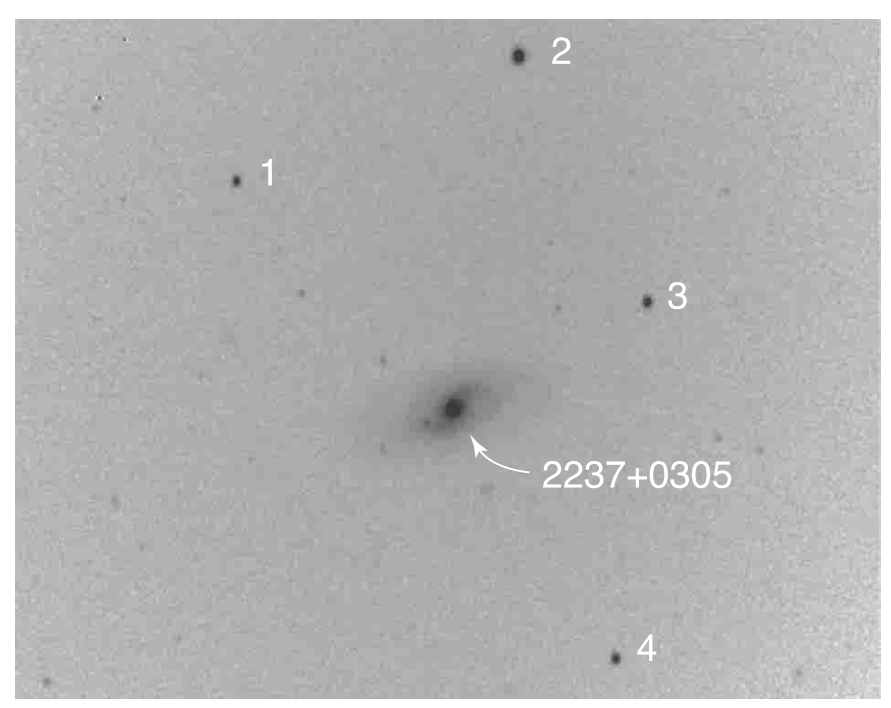

Fig. 2. Image of the environs of the lensing galaxy $2237+0305$ in the $r$-band. This image was composed from 10 images taken at APO on 20 November 1996. The four reference stars are marked 1 to 4 . The four quasar images are situated in the core of the galaxy. The image size is $4.2 \operatorname{arcmin} \times 2.9$ arcmin. North is down and east is to the left.

assumption that the two stars are not variable. If one or both of the stars were variable, our photometry would still be accurate to less than $0.02 \mathrm{mag}$ in $65 \%$ of the cases, and 0.04 mag in $95 \%$ of the cases. We use the $r$-magnitude determined by Corrigan et al. (1991, their star $\alpha$ ) for star $\# 3\left(m_{3}=17.28 \mathrm{mag}\right)$ and the magnitude difference $m_{3}-m_{2}$ determined above (with the described uncertainty) to obtain the $r$-band magnitude of star \#2 as $m_{2}=15.65 \mathrm{mag}$.

The frames of each night are added into a single image. The offsets between different frames are calculated from all reference stars. The frames are then aligned using the IRAF task "imalign". In this process, all frames are bilinearly interpolated to the positions of one reference frame;

3. The light distribution of the galaxy is modelled numerically using a model (Schmidt 1996) that was obtained from the analysis of an archival HST $I$-band image of the galaxy (Westphal 1992). The model consists of a de Vaucouleurs (1948) bulge and an exponential disk. The de Vaucouleurs brightness profile $b(r)$ is given by (in magnitudes per pixel):

$b(r)=2.5 \log i_{0}-3.33\left[\left(\frac{r}{r_{\text {bulge }}}\right)^{1 / 4}-1\right]$,

where $i_{0}$ is the central intensity (in counts per pixel) and $r_{\text {bulge }}=(4.1 \pm 0.4)$ arcsec is the half-light radius. Since the galaxy is inclined with respect to the line of sight, we use an elliptical surface brightness distribution for the bulge with a constant axis ratio of 0.69 and a position angle, indicating the galaxy inclination axis, of $77^{\circ}$ (measured from north through east). The exponential disk brightness profile in magnitudes per pixel is given by

$b(r)=2.5 \log i_{0}-\Delta b_{0}-\frac{r}{r_{\text {disk }}}$

with $r_{\text {disk }}=(11.3 \pm 1.2)$ arcsec and $\Delta b_{0}=(1.0 \pm$ $0.1) \mathrm{mag} \operatorname{arcsec}^{-2}$. Due to the galaxy inclination, the surface brightness distribution of the disk is seen with an axis ratio 


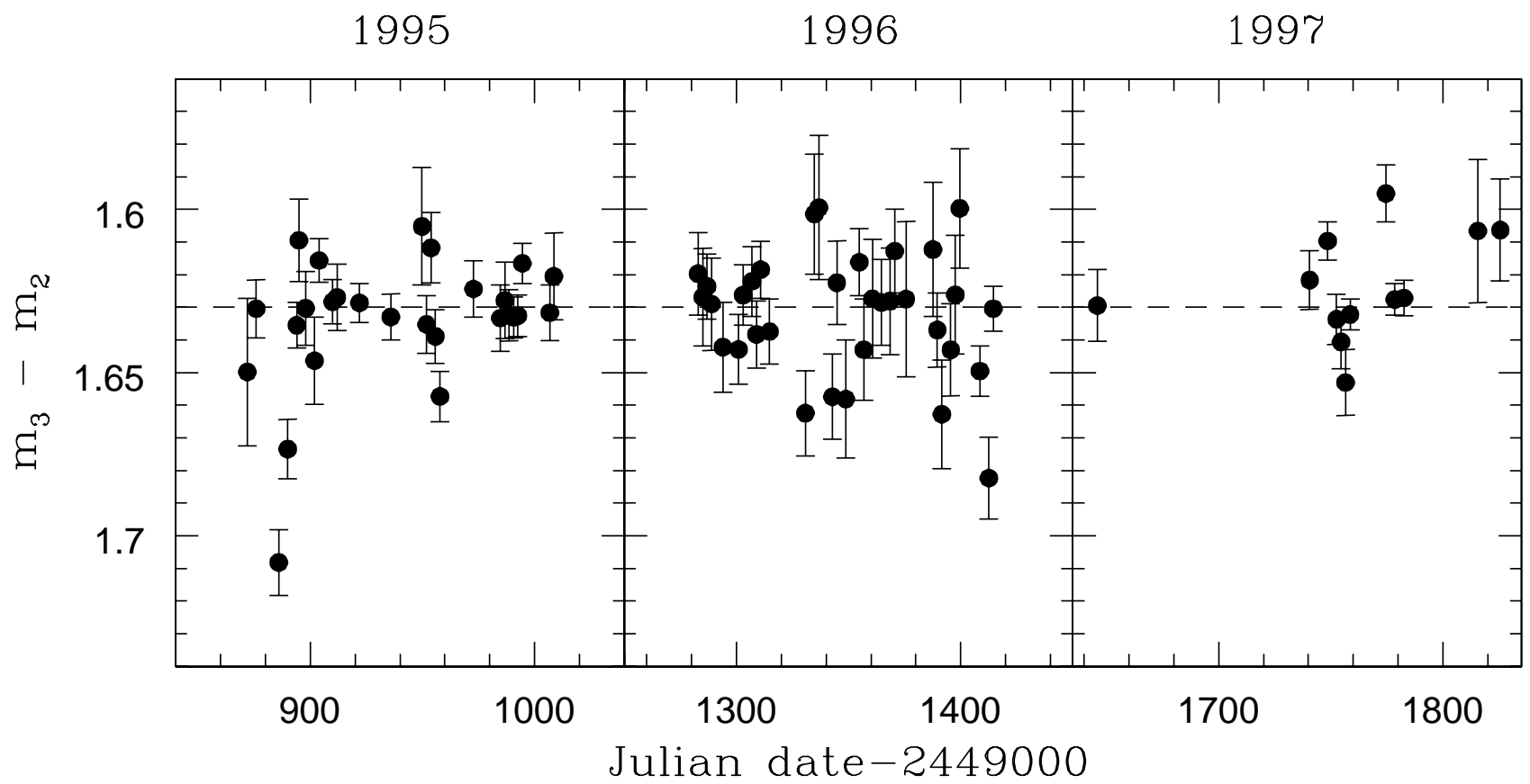

Fig. 3. Magnitude difference $m_{3}-m_{2}$ between reference stars \#3 and \#2 in the 73 combined frames. The Poisson errors of the star fluxes were added in quadrature. The median magnitude difference 1.63 mag is shown with a dashed line.

of 0.5. Although the Apache Point data were taken in the $r$-band, we only left the amplitude $b_{0}$ a free parameter and, for simplicity, kept the offset $\Delta b_{0}$ as measured using the HST image. The galaxy light distribution was subsampled by a factor ten in each direction before mapping it to the pixel grid of the detector. The decomposition of the galaxy brightness profile into different components is not unique. It is, for example, also possible to use two exponential profiles, which could in general even be a preferable solution (Andredakis \& Sanders 1994). However, any well-fitting description of the light distribution in the inner 3 arcsec of the lensing galaxy will be good enough for us because the differences are washed out by the coarse sampling of the data, the seeing at Apache Point of more than an arcsecond, and noise.

In Fig. 4, the central pixels of the frame from Fig. 1 are shown after the galaxy model has been subtracted. The remaining quasar images $\mathrm{A}$ and $\mathrm{B}$ (marked in the figure) are seen. Images $C$ and D cannot be detected with any significance. Some very faint remaining structure is seen around the quasar images that is due to the bar-like spiral arm structure that extends into the galaxy centre (Yee 1988; Schmidt et al. 1998) and that had not been included in the galaxy surface brightness model;

4. Given the seeing of our images, we consider the 5 mas accuracy of the Blanton et al. (1998) HST coordinates of the four quasar images relative to the galaxy centre as "exact". In the first fitting run, the galaxy position and amplitude, as well as the four quasar images are now fitted with the nonlinear minimization routine AMOEBA (Press et al. 1992). The quasar images are represented by four circular Gaussian brightness profiles with a full width at half maximum as determined from the seeing of the reference

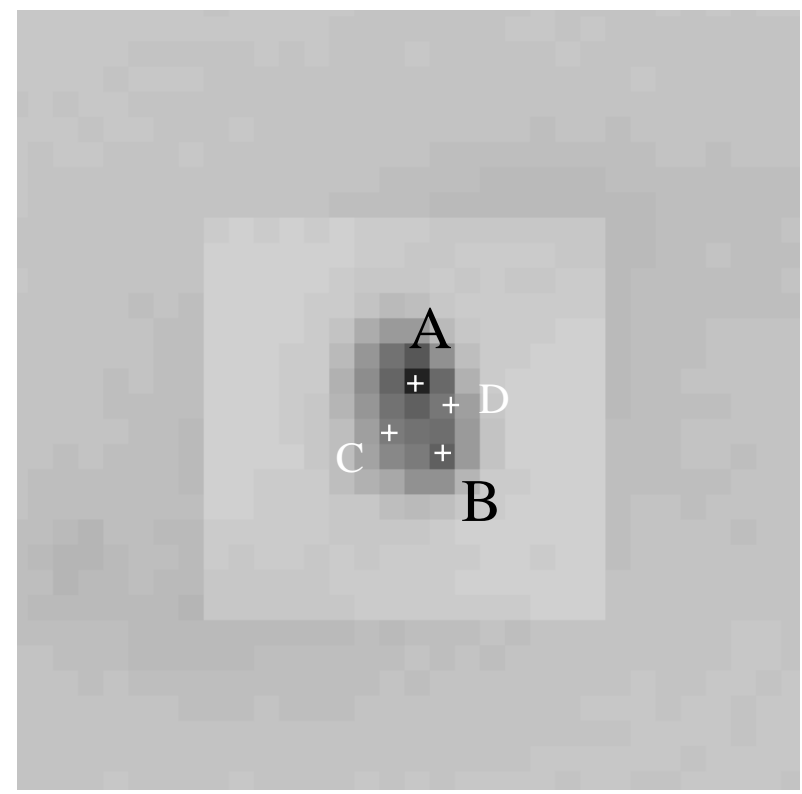

Fig. 4. Image of the central $(18.6 \mathrm{arcsec})^{2}$ of the lensing galaxy $2237+0305$ in the $r$-band. The light from the galaxy has been subtracted in the inner square with 9.6 arcsec (or 16 pixel) side length. This image was taken at APO on 20 November 1996 under 1.2" seeing. The raw image (before subtraction of the galaxy) is shown in Fig. 1. The plus signs mark the quasar image positions as determined from HST (Blanton et al. 1998). The two point sources in the middle are the quasar images $\mathrm{A}$ and $\mathrm{B}$. Images $\mathrm{C}$ and $\mathrm{D}$ are not detected with any significance because their amplitudes are not larger than the noise. North is down and east is to the left.

star. The Gaussian profiles were subsampled by a factor ten in each direction before mapping them to the pixel grid of the detector. The analytical galaxy model is also convolved 
with this Gaussian seeing model. AMOEBA is used to search for the best-fit solution in the parameter space of galaxy position, galaxy amplitude, and quasar fluxes (as determined by the amplitudes of the Gaussians) using a $\chi^{2}$ goodness of fit estimator over the central $9 \times 9$ pixels:

$\chi^{2}=\sum_{9 \times 9 \text { inner pixels }} \frac{\left(i_{\text {model }}-i_{\text {observed }}\right)^{2}}{i_{\text {observed }}}$.

In order to constrain the parameter search to relevant parts of the parameter space, we introduced additional constraints (priors) such that trespassings across certain limit values were added quadratically to Eq. (3). This is used to enforce the constraints that all fluxes are positive, and that the fluxes of image $\mathrm{C}$ and $\mathrm{D}$ are smaller than the fluxes of A and B. The latter assumption became wrong in 1998 and 1999 where image C "overtook" image B during a brightness peak beautifully resolved by OGLE. However, our data record stops in 1997, and until then there is no evidence for a significant rise of either image C or D towards the fluxes of A or B. As will be reported in Sect. 4, our flux estimates have also been quantitatively confirmed at a few points by independent observations;

5. In step 4 we have already produced a light curve with flux estimates for all quasar images. In this final step we use the results determined in step 4 for all 73 nights and determine the median galaxy amplitude and the median distances of the reference stars \#2, \#3, and \#4 from the centre of the galaxy.

The fitting procedure is then repeated with a fixed value for the galaxy amplitude. The galaxy position is also fixed by the distances to the three reference stars \#2, \#3, and \#4. Thus, only the four quasar fluxes are still free parameters. The distances of stars \#2, \#3, and \#4 from the galaxy centre are

$r_{2}=(95.09 \pm 0.19) \mathrm{arcsec}$,

$r_{3}=(64.55 \pm 0.12) \mathrm{arcsec}$,

$r_{4}=(81.59 \pm 0.12)$ arcsec.

( $1 \sigma$ error bars). The result of the fitting process is a best-fit model with parameters and uncertainties that can be estimated from the depth and steepness of the $\chi^{2}$-surface dip (Press et al. 1992).

We estimate additional uncertainties of the fluxes by varying the galaxy position within the range allowed by the separations of galaxy centre and reference stars. This is done by using 10 random positions for the galaxy allowed by the $1 \sigma$-error bars of $r_{2}, r_{3}$ and $r_{4}$ and by carrying through the whole analysis procedure. The error bars given in Table 1 and Fig. 5 are determined with this method.

\section{Results}

We obtain $r$-band light curves for images A and B as shown in Fig. 5, and detailed in Table 1. The fluxes of images $C$ and D are not measurable with our data since the images are lost in the noise.

Despite the large error bars of the order of $\approx 0.15$ mag for the individual data points in Fig. 5, there is evidence for a significant brightness peak in the light curve of image A in 1996 with an amplitude of about 0.4 to 0.5 mag (relative to 1995). Theoretical models of the system predict that intrinsic fluctuations of the quasar appear in images $\mathrm{A}$ and $\mathrm{B}$ with a timedelay of a few hours (Schneider et al. 1988; Wambsganss \& Paczyński 1994). Since image B does not vary much - there is some indication for a slight smooth decrease - the uncorrelated flux variation in image A can safely be interpreted as microlensing in the lensing galaxy.

Since we had to carry out an involved data reduction process to obtain photometry for the quasar images from the APO data, we used the $V$-band data points by Vakulik et al. (1997, crosses), Burud et al. (1998, stars) and the OGLE group (Woźniak et al. 2000a, triangles) for a comparison. Since the APO data have been taken in the $r$-band, these data points were plotted in Fig. 5 by making the assumption that the $r$-band magnitude of quasar image A on JD-2449000 $=1800$ is $m_{r}=17.3 \mathrm{mag}$. Assuming a simple additive colour correction, we derive the relation $m_{r}=m_{V}-0.05$ from the OGLE data for image A. For image B, we used $m_{r}=m_{V}-0.01$ in order to roughly account for the colour difference between the two quasar images. This value was determined using the $R$-band results from Vakulik et al. (1997) (Maidanak Observatory, 17/19 September 1995, JD-2 $449000=978 / 980)$ and Burud et al. (1998) (Nordic Optical Telescope (NOT), 10/11 October 1995, JD-2 $449000=1000 / 1001)$.

Overall there is rather good agreement between our data and these independent observations. In 1995, our results seem to favour a slightly fainter image B. However, those of our data points that are nearest in time to the independent measurements, agree within the error bars. It is unclear whether this could be due to short-duration small-scale microlensing, or a very small systematic offset. In 1996, both the NOT (Østensen 2001, priv. comm.) and Maidanak Observatory (Shalyapin 1999, priv. comm.) observed that image A had become much brighter than the other images.

We conclude that within the error bars we have quantitative confirmation for our measurements in 1995 and 1997 for a few selected epochs. In 1996 there is independent qualitative evidence for a brightness rise of image A.

\section{Discussion}

We have presented monitoring data of Q2237+0305 over a period from 1995 to early 1998. Although the error bars on individual data points are relatively large, the coverage of 73 nights clearly enables us to see some significant trends in the microlensing behaviour. In agreement with the results from the OGLE group (Woźniak et al. 2000a,b), we find that Q2237+ 0305 shows large magnitude variations of several tenths of a magnitude on timescales of less than hundred days.

It has become evident that the microlensing variations in Q2237+0305 can happen rather smoothly over time spans of several months to years. The Irwin et al. (1989) event (also in its interpretation by Racine (1992) as the first half of a double peak) seems to be an example of a short-duration microlensing variation. The Pen et al. (1993) drop is an example of a high-magnitude short-term process, but with very low signalto-noise at that time. 


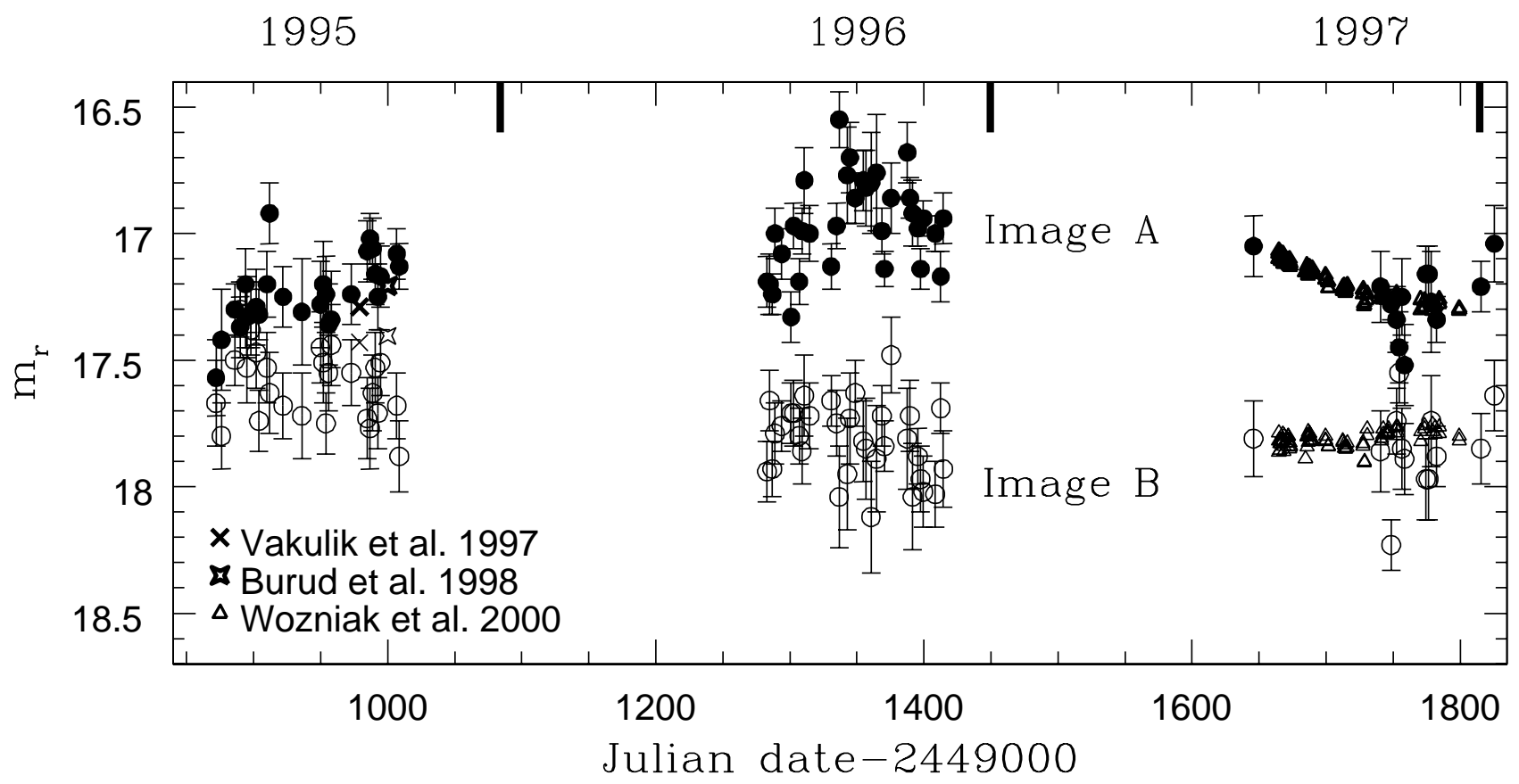

Fig. 5. Final $r$-band light curve for images A (filled circles) and B (open circles) of Q2237+0305 from June 1995 to January 1998. In the text, the epochs are referred to as 1995 (left), 1996 (middle), and 1997 (right). The start of each year is denoted by the thick markers at the top. The $V$-band data points by Vakulik et al. (1997, crosses), Burud et al. (1998, stars) and Woźniak et al. (2000a, triangles) are plotted with distinct symbols in this figure because the associated error bars are only 0.05 mag or less. To plot these symbols we assume that the plotted relative $r$-band magnitudes of image A can be obtained from the absolute $V$-band magnitudes via $m_{r}=m_{V}-0.05$. For image $\mathrm{B}$, we used $m_{r}=m_{V}-0.01$ to correct for the colour difference (see text).

All in all, it is fair to say that the observations of Q2237+0305 have started to resolve microlensing variations in great detail, and they look very much like the variations that were predicted already in the early $80 \mathrm{~s}$ for the microlensing effect. By filling up the gaps in the light curves, long-term monitoring programs pave the way for the statistical analysis of microlensing observations using, e.g., higher-order statistics of difference light curves (e.g., Kofman et al. 1997).

The OGLE light curve started where the data discussed in this paper stopped and thus shows the continuation of our light curve. At Apache Point, and at several other observatories (Maidanak Observatory, NOT), data are still being taken. A new detector is being used at APO that makes it possible to obtain much more accurate magnitude measurements for the quasar images (see, for example, the images at www . astro.princeton. edu/ ${ }^{\sim} \mathrm{elt} / 2237 . \mathrm{html}$ ).

Acknowledgements. We thank the referee P. R. Wozniak for his detailed and careful report and his very useful comments. We are grateful to Rainer Köhler for providing his library of FITS C-routines. RWS and UP thank the German-American Academic Council for sponsoring meetings in Aspen and Ringberg during which part of this work was done. ELT acknowledges support by the NSF grant AST98-02802. Part of this work was supported by the German Deutsche Forschungsgemeinschaft, DFG project number WA $1047 / 2-1$.

\section{References}

Andredakis, Y. C., \& Sanders, R. H. 1994, MNRAS, 267, 283

Blanton, M., Turner, E. L., \& Wambsganss, J. 1998, MNRAS, 298,
Burud, I., Stabell, R., Magain, P., et al. 1998, A\&A, 339, 701

Corrigan, R. T., Irwin, M. J., Arnaud, J., et al. 1991, AJ, 102, 34

de Vaucouleurs, G. 1948, Ann. Ap., 11, 247

Houde, M., \& Racine, R. 1994, AJ, 107, 466

Huchra, J., de Lapparent, V., Geller, M. J., et al. 1985, AJ, 90, 691

Irwin, M. J., Webster, R. L., Hewett, P. C., Corrigan, R. T., \& Jedrzejewski, R. I. 1989, AJ, 98, 1989

Kofman, L., Kaiser, N., Lee, M. H., \& Babul, A. 1997, ApJ, 489, 508

Lewis, G. F., Irwin, M. J., Hewett, P. C., \& Foltz, C. B. 1998, MNRAS, 295, 573

Østensen, R., Refsdal, S., Stabell, R., et al. 1996, A\&A, 309, 59

Pen, U., Howard, A., Huang, X., et al. 1993, in Gravitational lenses in the universe, ed. J. Surdej et al., Université de Liège, p. 111

Press, W. H., Teukolsky, S. A., Vetterling, W. T., \& Flannery, B. P. 1992, Numerical Recipes in C, second edition (Cambridge University Press, Cambridge)

Racine, R. 1992, ApJ, 395, L65

Schmidt, R. W. 1996, Master's Thesis, University of Melbourne

Schmidt, R., Webster, R. L., \& Lewis, G. F., MNRAS, 295, 488

Schneider, D. P., Turner, E. L., Gunn, J. E., et al. 1988, AJ, 95, 1619; Erratum 1988, AJ, 96, 1755

Vakulik, V. G., Dudinov, V. N., Zheleznyak, A. P., et al. 1997, AN, 318,73

Wambsganss, J. 1992, ApJ, 392, 424

Wambsganss, J., \& Paczyński, B. 1994, AJ, 108, 1156

Westphal, J. 1992, HST proposal ID 3799

Woźniak, P. R., Alard, C., Udalski, C., et al. 2000a, ApJ, 529, 88, see also www.astro.princeton.edu/ ogle/ogle2/ huchra.html

Woźniak, P. R., Udalski, A., Szymański, M., et al. 2000b, ApJ, 540, L65

Yee, H. K. C. 1988, AJ, 95, 1331 